\title{
An Integrated Optimization approach to locate the D-STATCOM in Power Distribution system to reduce the power loss and Total Cost
}

\author{
S. Muthubalaji ${ }^{1}$, R. Anand ${ }^{2}$, N.karuppiah ${ }^{3}$ \\ ${ }^{1,2}$ Professor, Department of Electrical \& Electronics Engineering, CMR College of Engineering \& Technology, Kandlakoya, \\ Hyderabad, Telangana. \\ ${ }^{3}$ Professor, Department of Electrical \& Electronics Engineering, Vardhaman College of Engineering, Shamshabad,Telangana
}

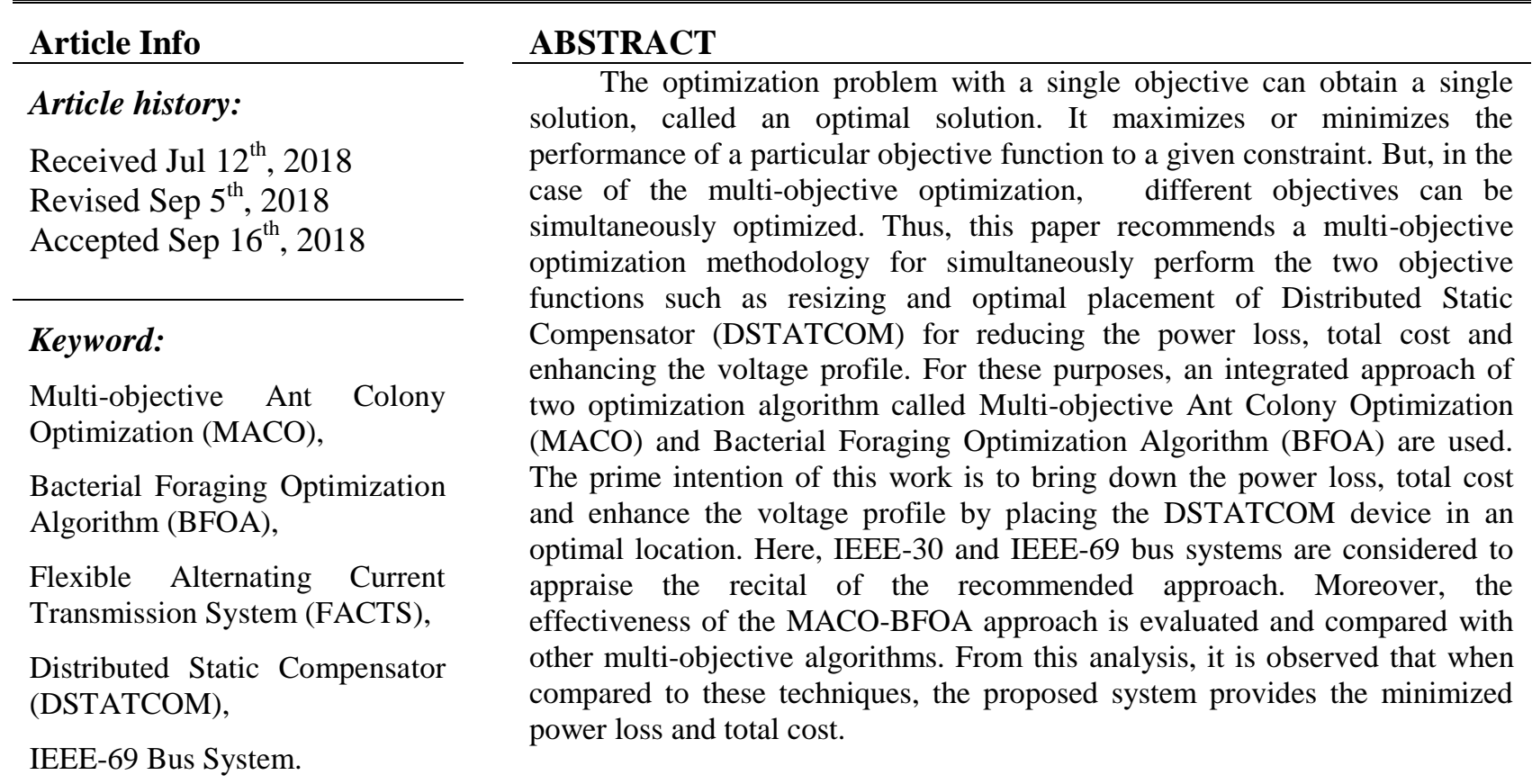

\section{Corresponding Author:}

S. MUTHUBALAJI,

Department of Electrical \& Electronics Engineering,

CMR College of Engineering \& Technology,

Kandlakoya, Hyderabad, Telangana

\section{Introduction}

Distribution is the procedure of transferring the electricity from generating station to the consumer premises. The transfer of power from generating plant to the consumer is carried through a transmission system and distribution system. In general, the distribution station is the part of the electric power system, which delivers the power to the customers for consumption. The electrical distribution is performed at a constant voltage level. Improving the power quality in a distribution system is an important consideration for reactive power compensation. For this purpose, the DSTATCOM [1]device is used in this work. This device is mainly used to solve the problems due to power quality issues and the unbalanced faults in the distribution network. DSTATCOM is a shunt type FACTS device that controls the magnitude as well as the phase angle. Here, the DSTATCOM is optimally placed in the IEEE- 69 bus system and the location is decided by the best fitness value for the power loss reduction.

The STATCOM consists of an IGBT based voltage converter for voltage control and for compensating the reactive power in power systems. This voltage source converter converts a DC voltage into an $\mathrm{AC}$ voltage which is used for active and reactive power compensation of the system. It is also, enhances the 
transient stability and controls the power flow. It gives many advantages as the fast response and higher voltage support capability. In this paper, an integrated algorithm namely, MACO-BFOA approach is proposed to optimally place and resize the DSTATCOM device for reducing the power loss[2]. In addition, the total expenditure on the overall distribution system is also minimized by reducing the power loss. The recommended MACO-BFOA approach is used to solve the multi-objective optimization problems. The intention of using this integrated methodology is to optimize the size of the DSTATCOM device for the reactive power generation in order to reduce the power loss. The ACO is applied for choosing the minimum cost path. Here, this approach is effectively applied for solving the single objective optimization problem. This type of problem minimizes or maximizes a particular objective, subject to a given constraint. BFOA is a type of computation algorithm developed based on the foraging nature of the E.Coli bacteria. In BFO algorithm, each bacterium tries to get sufficient amount of nutrient and to evade the unsafe substrates. Individual bacterium attempt to get the optimized solution for these two problems altogether and this behavior used for the multi-objective optimization. Furthermore, BFOA is one of the globally accepted optimization algorithms for distribution and control. Instead, the multi-objectives are distinct and are need to be optimized simultaneously. Here, the optimal placement of DSTATCOM is identified through the reliability indices SAIFI and SAIDI. The main intention of using SAIDI is to calculate the power and point out the error location in the distribution network.

The role of SAIFI is to find out the suitable location for minimal power loss and evaluate the best fitness value. Initially, the distributed network is formed and the link between the buses is established. The initial particles are assigned to the DSTATCOM and the sample weight for each particle is assigned by using the random weight value. Then, the SAIDI and SAIFI values are calculated based on this weight value. Here, the SAIDI value is used to resize the DSTATCOM device based on the following parameters: capacitive range and reactive power components. After updating the weight, the ant path is detected. If the link of the path is satisfied, the DSTATCOM is placed based on the best fitness value. Finally, the reactive power is generated to reduce the power loss. Here, the IEEE-69, IEEE-30 and IEEE-33 bus systems are taken to evaluate the performance of the proposed system. In this work, the integrated MACO-BFOA is compared with some of the existing algorithms such as Fuzzy-Genetic Algorithm (GA), Fuzzy-Particle Swarm Optimization (PSO), Fuzzy-Ant Colony Optimization (ACO) and Non-dominated Sorting Genetic Algorithm (NSGA). When compared to these techniques, the proposed MACO-BFOA provides the minimized power loss and total cost.

This paper is organized as follows: Section II involves the existing works related to optimal placement and resizing of DSTATCOM in power distribution systems. Section III discusses the comprehensive explanation of the proposed method: MACO-BFOA. Section IV deals the simulation results and parameters of the proposed system. Finally, this paper is concluded in Section V

2.

Related Work

In this part, the already used technique, algorithms have been proposed for placing and resizing of DSTATCOM for minimizing the power loss and overall cost of the power distribution systems are discussed.

In [3] the method of placing the STATCOM device which is tuned genetically to maintain voltage stability and for reactive power management in the distribution system. The STATCOM is designed to maintain the voltage regulation. In this study, GA is applied to select the optimum value of capacitor and battery sources. Mohanty et.al [4] recommended the Flexible AC Transmission device to advance the power system stability in the distribution system. Here the power system steady state control is achieved by FACTS controllers and it draws the advantages of increased reliability as well as the availability of the transmission system. Rohit, et al [5] reviewed the role of STATCOM for power quality improvement. STATCOM is most suitable to solve the congestions problem in transmission lines. In [6] the author suggested the FACTS devices diminish the power quality problem like voltage sag in distribution systems. Even though FACTS devices were most suitable for transmission networks, related ideas are applied in distribution systems.

Solmon, et al[7] presented an integrated approach of GA and ACO to decipher multi-objective optimization task. In this work, the optimal solution depends on the parameters like a number of ant colonies and the number of pheromone trails. Moncayo, et al [8] proposed a distinctive multi objective approach called Pareto ACO algorithm to find out the Supply Chain (SC) design for complex hierarchies of subassemblies and components. In this [14] paper the network configuration problem was addressed by an efficient algorithm namely, Fireworks Algorithm (FA). The reconfiguration of the network was done to reduce the power loss and improve the voltage stability. In [9] the author used ACO and DE algorithm to optimize the reactive power in 
distribution systems. Akorede, et al[10] presented a brief survey of the existing techniques which are available to find the optimal location DG in Electric power distribution systems. In this survey, the ACO algorithm was recommended to solve the optimization problem under consideration.

Sathish Kumar et.al [11] proposed a fast and effective $\quad$ BFO algorithm to reduce the power loss in the power distribution network. Moreover, the proposed approach was extremely appropriate for Distribution Automation System. Kumar et.al [12] reduce the power loss and load balancing index through reconfiguration. The BFO algorithm was suggested to obtain reconfigured without affecting the radial structure with minimal loss. In this[13] the author, device the power system stabilizer by using the BFOA algorithm. The Power System Stabilizer (PSS) control the oscillations in power system and maintain the stability. In this paper, the main role of BFOA was to minimize the execution time by selecting the suitable controller parameters. The PSS was designed in such a way to neutralize the oscillations in the multi-machine power system.

Vivekananthan et al[14] proposed an efficient technique to reduce the power loss and to improve the voltage stability by using the optimization technique called BFOA. To achieve the task the author selected three different FACTS devices namely, Static VAR Compensator (SVC), Thyristor Controlled Series Capacitor (TCSC) and Unified Power Flow Compensator (UPFC) for the improvement of voltage stability. An advanced particle swarm optimization technique namely, Fully Informed Particle Swarm Optimization (FIPSO) suggested in [15] to minimize the power loss in the power Transmission system. In this paper, the author has considered different transmission systems like Ward-Hale-6-Bus system, IEEE 30-system, and IEEE 118-bus system. Naik, et al[16] suggested an approach to reduce the real power loss in the electrical distribution system. In this approach, a sensitivity analysis was used to spot the optimal locations for DG and capacitor. In [17] the author advice a mathematical model to reduce the power losses on transmission systems.

Kayal et. al [18] proposed a multi-objective Particle Swarm Optimization algorithm for power loss minimization and voltage stability improvement in the radial distribution system. Here the voltage stability levels are estimated by a Voltage Stability Factor (VSF) which is very simple and efficient tool for the stability level calculation. In [19] an online Optimization Strategy was introduced for the minimization of the power losses throughout the feeder in power systems. The effectiveness of power loss reduction is witnessed by the numerical results. Duan, et al [20] proposed an improved Genetic Algorithm (GA) for power loss diminution and reliability enrichment in distribution networks through reconfiguration of the network. The efficacy of this technique was tested in 33-bus, 69-bus, and 136-bus radial distribution systems. Moradi and Abedini [21] proposed a hybrid GA and PSO algorithms for sitting and sizing of DG to minimize the power losses and improve the voltage stability in the distribution network.

From the study of the existing methodology, it clearly reveals that the practiced methods are not very effectively play role in power loss reduction and cost-effectiveness. In order to surmount these drawbacks, an integrated MACO- BFOA approach is suggested in this paper. When appraised of the existing methods, the proposed approach can solve the problem of power loss reduction, cost minimization and reliability enhancement in a better way.

\section{Proposed MACO-BFOA Approach}

This section presents the detailed explanation of the integrated MACO-BFOA approach. In this paper, the combined MACO-BFOA approach is proposed for optimal placement and resizing of DSTATCOM in power distribution system. Fig 1 describes the overall flow of the proposed MACO-BFOA approach. At first, the distribution network is designed and the power is extracted. Then, the features i.e. branches are formed to find the faulty condition and the initial particles are assigned based on the DSTATCOM device. The feature parameters such as bus system power, base angle and voltage for each time sampling are included in each branch. Hence, the sample weight for each particle is assigned. Based on this value, the SAIDI and SAIFI values are calculated and these values are used to perform the weight updation. Here, the SAIDI value is used to resize the DSTATCOM. Then, the present weight values are compared with previous values, if the present weight is greater than the previous weight, the weight value will be updated; otherwise, the previous weight value will be taken. Based on this updation, the path of the ant is detected. If the path link is satisfied, the DSTATCOM will be replaced in that place by using the best fitness value. Hence, the reactive power is generated to minimize the power loss in the electrical distribution network. 


\subsection{Multi-objective Optimization Algorithm MACO-BFOA}

An optimization problem with multiple objectives is known as multi-objective optimization algorithm. In this system, the two main objectives are taken to bring down the power loss, total cost and to enhance the voltage profile. The objectives of this work are shown in below:

- $\quad$ Resizing of DSTATCOM

- $\quad$ Optimal placement of DSTATCOM

The MACO-BFOA algorithm is also used to achieve the best system reliability while concurrently reduces the total cost of the system. In this system, the objective functions are multi-dimensional and have the solution of partial order. Due to the nature of the distribution network, some modifications are done to keep the radial structure which reduces the searching requirement. When compared to other multi-objective algorithms, the MACO-BFOA algorithm provides the best optimization results.

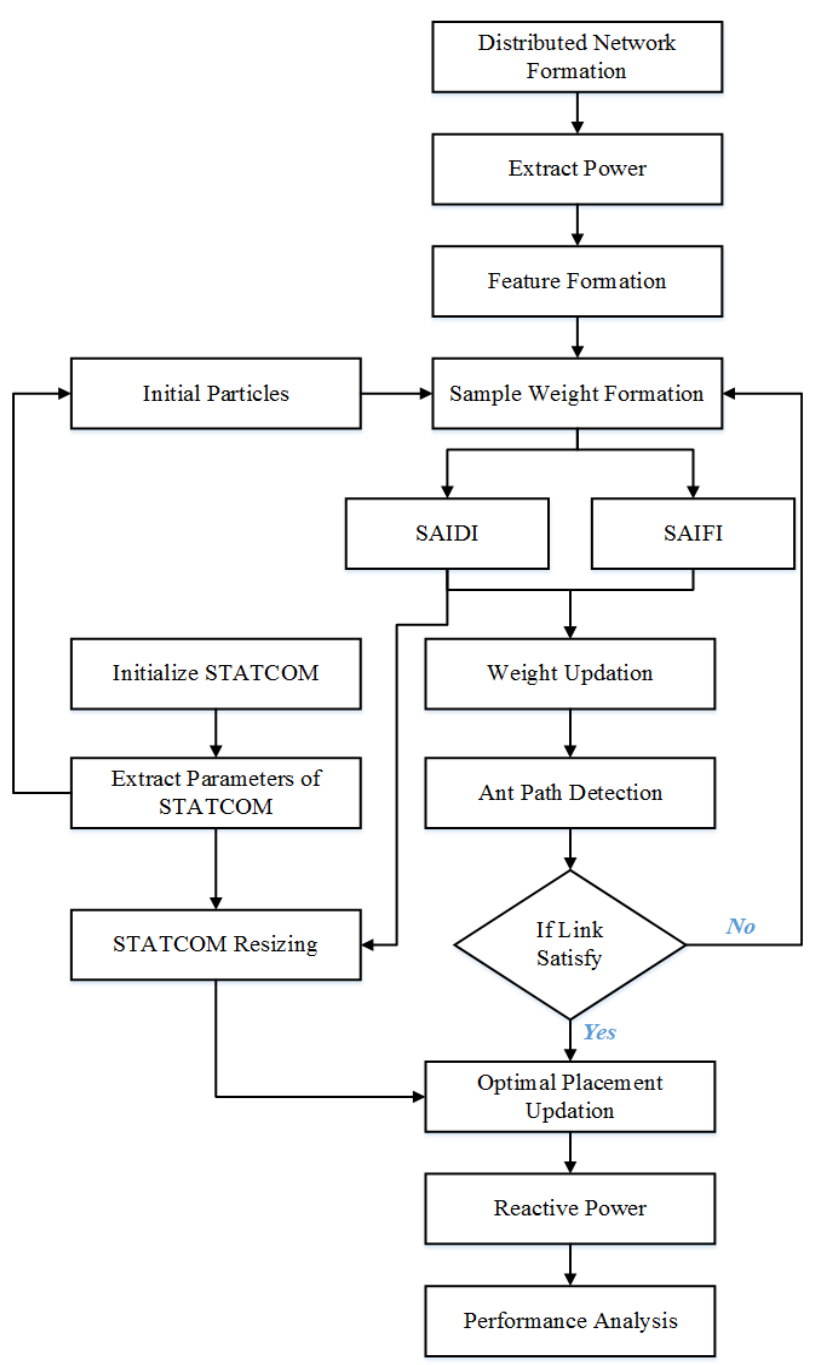

Fig 1 . The overall flow of the proposed system

\subsection{Resizing of DSTATCOM}

A low voltage distribution system is considered the STATCOM is identified as DSTATCOM. It is parallel connected power electronics based FACTS device, which facilitates the reactive power support to a bus. It is mainly intended to generate or absorb the reactive power. The major components of the DSTATCOM are Alternate Current (AC) filter, Direct Current (DC) capacitor, IGBT and coupling transformer. It also contains a voltage source converter supplied by an energy storage device and it is connected to the distribution feeder. In this paper, SAIDI and SAIFI are used to calculate the weight value. 
- $\quad$ SAIDI

SAIDI is one of the most widely used indices to measure the utility value. Here, the DSTATCOM resizing is performed based on the SAIDI value.

- SAIFI

SAIFI is used to measure the effect of the power outage and also used to present the average frequency at which sustained interruptions occurred per customer. It is an indicator of utility network performance.

The calculation SAIDI and SAIFI values are shown in Algorithm I. Here, the SAIDI and SAIFI values are analyzed to choose the optimal placement of DSTATCOM in the distribution power networks.

\subsection{Optimal Location of DSTATCOM}

The optimal location and the quantity of reactive power generated by the DSTATCOM is the very important stage in DSTATCOM designing. Fig 2 illustrates the IEEE-69 bus system. Placement of DSTATCOM at this bus results in optimal reactive power reinforcement for improving the voltage profile. When compared to other optimal placement techniques, the amount of generated reactive power is improved by using MACO-BFOA. So, the loss of power occurred in the system is reduced.

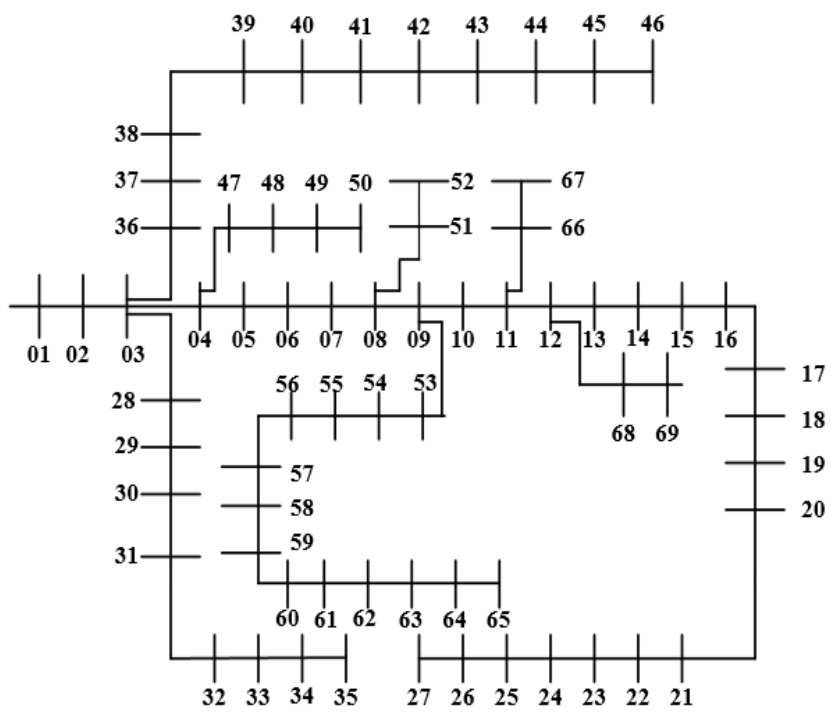

Fig 2. IEEE-69 Bus Model

Simulation results on a 69-bus of the IEEE are investigated, which is one of the well-known systems of the power quality. After simulation, the DSTATCOM is optimally placed and the reactive power is calculated by using a combination of MACO-BFOA.

\subsection{MACO-BFOA Algorithm}

At first, the parameter values are initialized. Then, the power is extracted from each bus location PW and the random particle is initialized based on the size of bacteria. Hence, the position of the population is evaluated with respect to the power input. Then, update the maximum area of population position and estimate the interruption of particles by calculating the SAIDI and SAIFI values. To achieve the required reactive power, the DSTATCOM device is resized with the minimum SAIDI value by changing the component parameters such as capacitive range and switching size. Then, the probability of minimum interruption particle is extracted with the help of SAIDI and SAIFI values. The ant path is detected and updated for the size of particles. The maximum best position of particles from the updated graph and the bus index value is extracted. Finally, the DSTATCOM device is placed in the 69-bus system for generating the reactive power.

\section{Step by step procedure for proposed algorithm}

Step 1: Initialize the parameters,

$$
p=2 ; \quad \text { // Dimension of search space }
$$




$$
\begin{array}{cl}
s=26 & \text { // No of bacteria } \\
N c=50 ; & \text { // No of chemotactic steps } \\
N s=4 ; & \text { // Limits the length of a swarm } \\
\text { Nre }=4 ; & \text { // No of reproduction steps } \\
\text { Ned }=2 ; & \text { // No of elimination dispersal events } \\
\text { Sr }=\text { s/2;//No of bacteria reproductions per generation } \\
\text { Ped }=0.25 ; \quad \text { // Probability of eliminated bacteria } \\
\text { Amin }=0.5 ; & \text { // Minimum area limitation } \\
\text { Amax }=1.5 ; & \text { // Maximum area limitation }
\end{array}
$$

Step 2: Extract the power from each bus location, $P W$

Step 3: Initialize random particles for the size of bacteria,

Step 4:

$$
\text { Particle }=\text { Random }(1 \text { to } s)
$$

$$
\begin{aligned}
& \text { Position }=p_{1} * q_{1}+\left(x+A_{\max }\right)^{2}+\left(y+A_{\min }\right)^{2} \\
& p_{1}=\sum_{k=1}^{N C}(k * \cos ((k+1) * x+k)) \\
& q 1=\sum_{k=1}^{N C}(k * \cos ((k+1) * y+k))
\end{aligned}
$$

Where, $x$ and $y$ represents the coordinates of particle location.

Step 5: For the length of swim Ns,

Update maximum area of population position and update population as

If (the position is updated)

$$
\text { Position }= \begin{cases}\text { Position }_{\text {Last }} & \text { if Position } \\ & \text { else Post Position } \\ \text { present }\end{cases}
$$

End if;

Update the population as in step 3 and 4 in the range of Nre for each user;

If (The updated particle < Ned)

End if;

Eliminate the particle;

End loop;

Step 6: Estimate the interruption of particles by using,

$$
\begin{aligned}
S A I F I & =\frac{\text { Number of particles in minimum weight }}{\text { Total number of particles alive }} \\
& =\frac{\left(\sum\left(\left(\text { Particle }_{i}\right) *\left(\text { Nval }_{i}\right)\right)\right)}{\left(\sum\left(\text { Nval }_{i}\right)\right)} \\
S A I D I & =\frac{\text { Sum of particles iteration for a minimum weight }}{\text { Total number of particles alive }} \\
& =\frac{\left(\sum\left(\left(\text { Duration }_{i}\right) *\left(\text { Nval }_{i}\right)\right)\right)}{\left(\sum\left(\text { Nval }_{i}\right)\right)}
\end{aligned}
$$

Where Particles represents minimum weight value, $N_{v a l}$ indicates the number of particles in the iteration and Duration defines the number of iteration at particle failure.

Step 7: Resize the DSTATCOM with minimum SAIDI value by changing the component parameters for achieving required reactive power.

Step 8: Extract the probability of minimum interruption particle by using the SAIDI and SAIFI values.

Step 9: Estimate the graph and path by using,

Path $=$ Probability $_{\text {minimum }}($ Particles at $p)$;

Graph $=$ position of particles;

Step 10: Update the path

For 1 to the size of particles

$$
\begin{array}{ll}
\text { If }(\text { path }>\text { Ped }) \\
\text { Path }_{\text {update }}
\end{array}= \begin{cases}\text { Graph }>\text { Path } & \text { Graph } \\
\text { else } & \text { path }\end{cases}
$$

Else

Loop to step 4;

End if;

Step 11: Extract the maximum best position of particles from the updated path 
Step 12: Extract the bus index value

Step 13: Place the DSTATCOM device

Step 14: Finally, generate the reactive power

\section{RESULTS AND DISCUSSION}

This part investigates the simulation results of the IEEE 69-bus system for calculating the reactive power by using the MACO-BFOA approaches. The simulation DSTATCOM is carried out by using the MACO-BFOA approach for power loss minimization and total cost minimization.

An Optimal location of DSTATCOM in IEEE-30 bus systems

Fig 3 illustrates the maximum value of fitness function for various buses obtained by applying MACO-BFOA method. From Fig 3, it is observed that the optimal location for DSTATCOM in IEEE-30 bus system is number 8 . Table 1 describes the optimal placement location of DSTATCOM in IEEE-33 bus system.

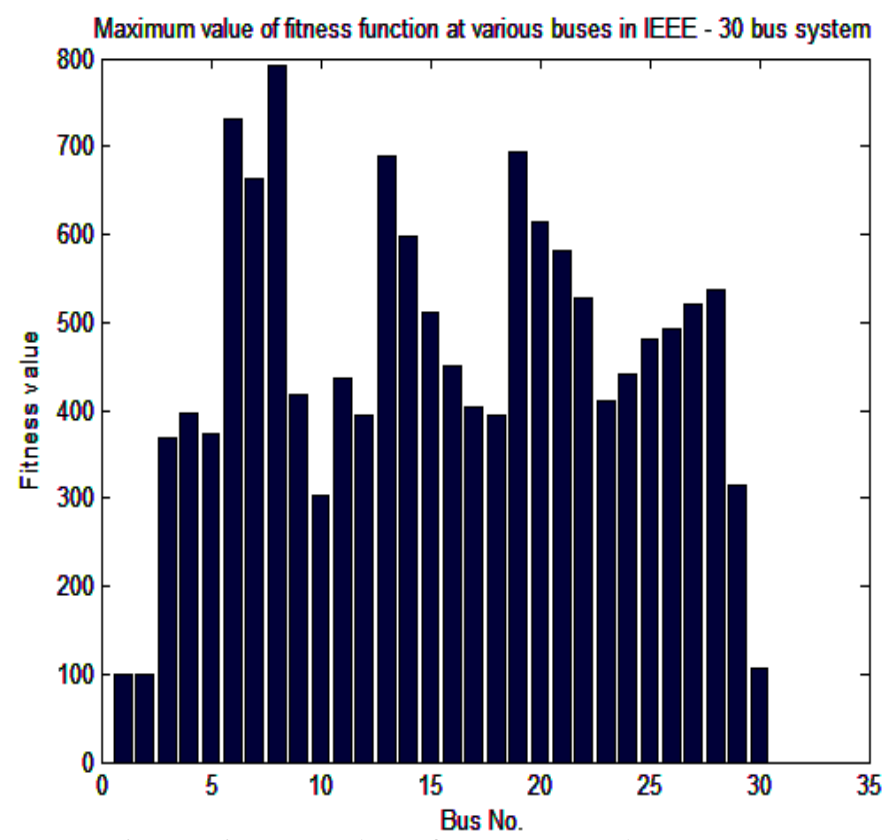

Fig 3. Fitness values for IEEE 30-bus system

Table 1. Optimal placement location of DSTATCOM for existing and proposed methods in IEEE-30 bus system

\begin{tabular}{|c|c|c|}
\hline \multicolumn{3}{|c|}{ IEEE-30 Bus System } \\
\hline Methods & DSTATCOM (MVA) & $\begin{array}{c}\text { Optimal Placement of } \\
\text { DSTATCOM }\end{array}$ \\
\hline Fuzzy-GA & 0.69 & 9 \\
\hline Fuzzy-PSO & 0.72 & 23 \\
\hline Fuzzy-ACO & 0.74 & 10 \\
\hline $\begin{array}{c}\text { Proposed Approach } \\
\text { MACO-BFOA }\end{array}$ & 0.76 & 8 \\
\hline
\end{tabular}

B. Optimal location of DSTATCOM in IEEE-69 bus systems 
Fig 4 illustrates the fitness values for various buses in IEEE-69 bus system. In this system, the DSTATCOM device is placed in bus-51.

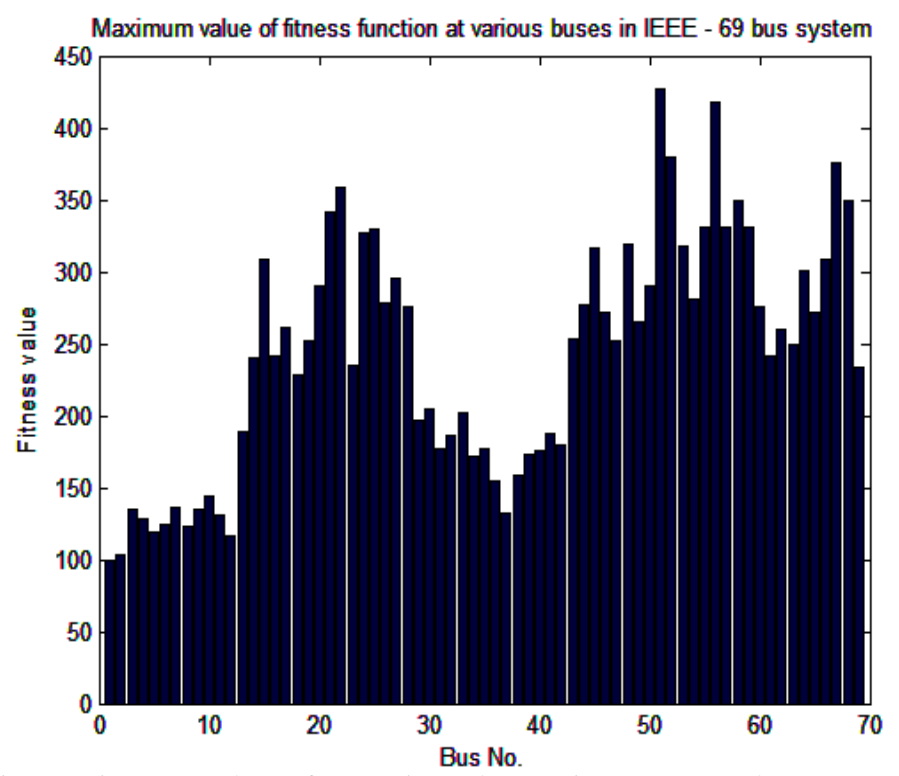

Fig 4. Fitness values for various buses in IEEE 69-bus system

Fig 5 represents the optimal placement of DSTATCOM in IEEE-69 bus system, where the device is placed in bus number 51 . Because the bus number 51 has the maximum fitness value and it generates more amount of reactive power. Thus, the DSTATCOM device is placed in bus-51.

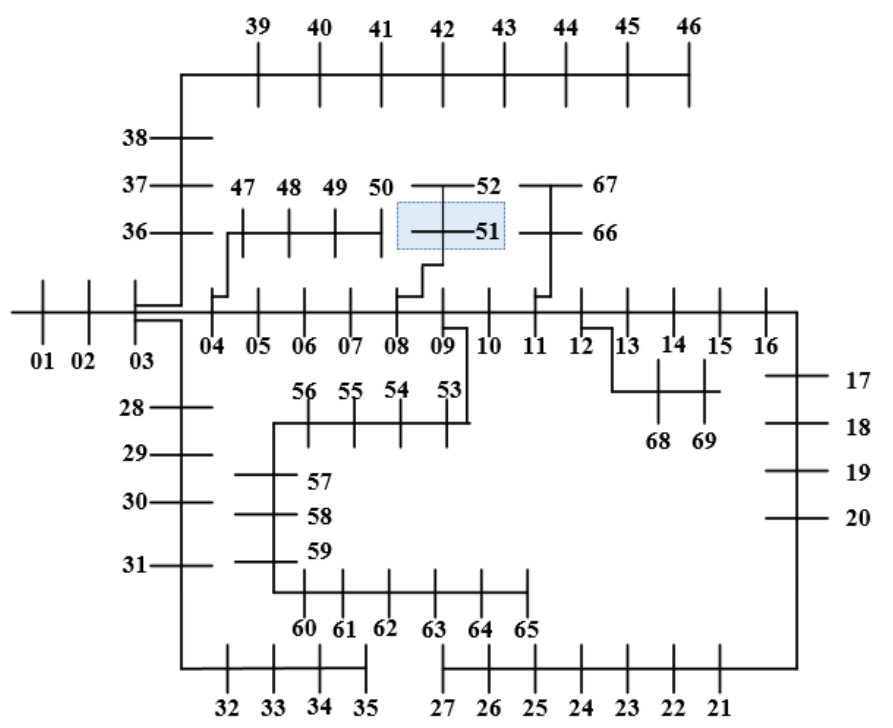

Fig 5. IEEE-69 Bus system with optimally placed D-STATCOM

\section{Performance Analysis of IEEE 30-bus System}

The results obtained in terms of power loss, voltage profile and load balance for existing and proposed methods are shown in Table 1. In this work, different multi-objective optimization algorithms[22] such as Fuzzy-GA, Fuzzy-PSO, Fuzzy-ACO are compared with the proposed MACO-BFOA algorithm. From this comparison, it is analyzed that the proposed method provides the low power loss, enhanced Voltage Profile (VP) and increased Load Balance (LB). The loss of power is calculated as follows:

$$
\text { Power Loss }=\sum_{j=1}^{f} \sum_{b=1}^{s} X_{b}\left|I_{b}{ }^{2}\right|
$$


Where $f$ defines the total number of feeders, $s$ defines the number of sections in the system, $I_{b}$ represents the current passing through line $b$ and $X_{b}$ is the resistance of the line section between buses $b$ and $\mathrm{b}+1$. The VP is calculated as follows:

$$
\text { Voltage Profile }(V P)=\sum_{j=1}^{f} \sum_{b \in l b}\left|V_{b}-V_{\text {ref,b }}\right|
$$

Where $\mathrm{lb}$ represents the collection of load buses, $\mathrm{Vb}$ defines the voltage amplitude at bus $\mathrm{b}$ and Vref, $\mathrm{b}$ is the nominal voltage at load bus b. The LB is calculated as follows:

$$
\text { Load Balance }(L B)=\sum_{j=1}^{f} \sum_{b=1}^{S}\left(\frac{I_{b}}{\frac{1}{f} \sum_{b=1}^{f} I_{b}}\right)^{2}
$$

Table 1. Power loss, voltage profile and load balancing values for the existing and proposed system in IEEE-30 bus system

\begin{tabular}{|c|c|c|c|}
\hline \multicolumn{4}{|c|}{ IEEE-30 Bus System } \\
\hline Methods & P_Loss $(\boldsymbol{k W})$ & $\boldsymbol{V P}$ & LB \\
\hline Fuzzy-GA & 73.26 & 0.87 & 48.59 \\
\hline Fuzzy-PSO & 69.15 & 0.84 & 45.67 \\
\hline Fuzzy-ACO & 48.73 & 0.82 & 43.03 \\
\hline $\begin{array}{c}\text { Proposed Approach } \\
\text { MACO-BFOA }\end{array}$ & 40.18 & 0.79 & 40.2 \\
\hline
\end{tabular}

The active power, reactive power and active power loss for existing[23] and proposed methods in IEEE-30 bus system are shown in Table 3. When compared to Trust-Region Sequential Quadratic Programming (TRSQP) method, the proposed MACO-BFOA approach provides the minimized power loss.

Table 3. Active power, reactive power and active power loss for TRSQP and MACO-BFOA approaches in IEEE-30 bus system

\begin{tabular}{|c|c|c|c|}
\hline \multicolumn{4}{|c|}{ IEEE-30 Bus System } \\
\hline Methods & $\begin{array}{c}\text { Active Power } \\
(\boldsymbol{M W})\end{array}$ & $\begin{array}{c}\text { Reactive Power } \\
(\text { MVar })\end{array}$ & $\begin{array}{c}\text { Active Power Loss } \\
(\mathbf{M W})\end{array}$ \\
\hline TRSQP & 3.715 & 2.3 & 0.203 \\
\hline MACO-BFOA & 2.31 & 2.8 & 0.152 \\
\hline
\end{tabular}

\section{Performance Analysis of IEEE-69 Bus System}

The active power, reactive power and active power loss for existing and proposed methods in IEEE69 bus system are shown in Table 4.The IEEE-69 bus system is used to test the performance of the proposed method. From this analysis, it is evaluated that the proposed system provides the minimized power loss in IEEE-69 bus system also.

Table 4. Active power, reactive power and active power loss for TRSQP and MACO-BFOA in IEEE-69 bus system

\begin{tabular}{|c|c|c|c|}
\hline \multicolumn{5}{|c|}{ IEEE-69 Bus System } \\
\hline Methods & $\begin{array}{c}\text { Active Power } \\
(\boldsymbol{M W})\end{array}$ & $\begin{array}{c}\text { Reactive Power } \\
(\text { MVar })\end{array}$ & $\begin{array}{c}\text { Active Power Loss } \\
(\text { MW })\end{array}$ \\
\hline TRSQP & 3.802 & 2.695 & 0.227 \\
\hline MACO-BFOA & 2.57 & 3.14 & 0.187 \\
\hline
\end{tabular}


D $\quad$ Simulation Results of IEEE-30 bus system for Total cost reduction

The total cost required for the circuit design is shown in Table 5. Here, the proposed MACO-BFOA technique is compared with the existing [24]Particle Swarm Optimization (PSO), Covariance Matrix Adapted Evolution Strategy (CMAES), Primal Dual Interior Point (PDIP) methods. From this, it is observed that the proposed method provides the minimized total cost when compared to these techniques. The IEEE-30 bus system is used for this cost analysis.

Table 5. Total cost for existing and proposed methods

\begin{tabular}{|c|c|}
\hline \multicolumn{2}{|c|}{ IEEE-30 Bus } \\
\hline Methods & Total Cost $\left(\mathbf{}^{*} \mathbf{1 0}^{\wedge} \mathbf{6}\right)$ \\
\hline PSO & 276.7 \\
\hline PDIP (Current Injection Model) & 79.98 \\
\hline CMAES & 79.32 \\
\hline PDIP (Transformer Model) & 75.532 \\
\hline CMAES & 74.429 \\
\hline MACO-BFOA & 71.205 \\
\hline
\end{tabular}

\section{Conclusions}

In this paper, an integrated approach of MACO-BFOA is proposed to optimally locate and resize the DSTATCOM device in a circuit for reducing the power loss and total cost. The proposed MACO-BFOA is a multi-objective algorithm that simultaneously performs two objective functions. At first, the initial particles are assigned based on the DSTATCOM and the sample weight is assigned for each particle. Based on this value, the SAIDI and SAIFI values are calculated and these values are used to perform the weight updation. Here, the SAIDI value is used to resize the DSTATCOM. Hence, the previous and present weight values are compared, if the present weight is greater than the previous weight, the weight will be updated; otherwise, the previous weight value will be taken. Based on this updation, the ant path is detected. If the path link is satisfied, the DSTATCOM will be placed in that location. Here, the optimal location of DSTATCOM is identified by the integrated approach of MACO-BFO. In this paper, the standard IEEE-30 and IEEE-69 bus systems are considered to test the performance of the proposed approach. Moreover, different existing multiobjective algorithms such as Fuzzy-GA, Fuzzy-PSO, Fuzzy-ACO, TRSQP, PDIP, and CMAES are compared with the proposed MACO-BFOA. The results obtained by simulation show that the proposed method provides the minimized power loss and total cost when compared to these techniques.

In future, the FACTS device with Distributed Generator (DG) will be optimized by using an efficient controller in order to reduce the total cost and load regulation in power system.

\section{References}

[1] A. Mahyavanshi, et al., "Reactive power compensation by controlling the DSTATCOM," International Journal of Emerging Technology and Advanced Engineering, vol. 2, pp. 212-218, 2012.

[2] A. Bagherinasab, et al., "Optimal Placement of D-STATCOM Using Hybrid Genetic and Ant Colony Algorithm to Losses Reduction," International Journal of Applied Power Engineering (IJAPE), vol. 2, pp. 53-60, 2013.

[3] N. Goel, et al., "Genetically Tuned STATCOM for Voltage Control and Reactive Power Compensation," International Journal of Computer Theory and Engineering, vol. 2, pp. 1793-8201, 2010. 
[4] Mohanty, Alok Kumar, and Amar

Kumar Barik. "Power system stability improvement using

FACTS devices." International Journal of Modern Engineering Research (IJMER) vol. 1, issue.2, pp. 666-672, 2011

[5] R. C. Rohit and K. K. Sharma, "Applications of STATCOM for Power Quality Improvement-A Literature Survey," International Journal of Recent Research Aspects, vol. 1, 2014.

[6] A. K. Goswami, et al., "Minimization of voltage sag induced financial losses in distribution systems using FACTS devices," Electric Power Systems Research, vol. 81, pp. 767-774, 2011.

[7] Alaya, Ines, Christine Solnon, and Khaled Ghedira. "Ant colony optimization for multiobjective optimization problems." Tools with Artificial Intelligence, 2007. ICTAI 2007.

19th IEEE International Conference on. Vol. 1. IEEE, 2007.

8] L. A. Moncayo-Martínez and D. Z. Zhang, "Multi-objective ant colony optimisation: A meta-heuristic approach to supply chain design," International Journal of Production Economics, vol. 131, pp. 407420, 2011.

[9] Y. Zhao, et al., "Distribution Network Reactive Power Optimization Based on Ant Colony Optimization and Differential Evolution Algorithm," Journal of Energy and Power Engineering, vol. 5, pp. 548-553, 2011.

[10] M. Akorede, et al., "A review of strategies for optimal placement of distributed generation in power distribution systems," Research Journal of Applied Sciences, vol. 5, pp. 137-145, 2010.

[11] K. Sathish Kumar and T. Jayabarathi, "Power system reconfiguration and loss minimization for a distribution system using bacterial foraging optimization algorithm," International Journal of Electrical Power \& Energy Systems, vol. 36, pp. 13-17, 2012.

[12] K. S. Kumar and T. Jayabarathi, "A novel power system reconfiguration for a distribution system with minimum load balancing index using bacterial foraging optimization algorithm," Frontiers in Energy, vol. 6, pp. 260-265, 2012.

[13] S. Abd-Elazim and E. Ali, "Optimal PSS Design in a Multimachine Power System via Bacteria Foraging Optimization Algorithm," WSEAS Transactions on Power Systems, vol. 8, 2013.

[14] J. Vivekananthan and R. Karthick, "Voltage Stability Improvement and Reduce Power System Losses by Bacterial Foraging Optimization Based Location of Facts Devices," International Electrical Engineering Journal (IEEJ), vol. 4, pp. 1034-1040, 2013.

[15] H. Tehzeeb-Ul-Hassan, et al., "Reduction in power transmission loss using fully informed particle swarm optimization," International Journal Of Electrical Power \& Energy Systems, vol. 43, pp. 364$368,2012$.

[16] S. Gopiya Naik, et al., "Optimal allocation of combined DG and capacitor for real power loss minimization in distribution networks," International Journal Of Electrical Power \& Energy Systems, vol. 53, pp. 967-973, 2013.

[17] O. M. Bamigbola, et al., "Mathematical modeling of electric power flow and the minimization of power losses on transmission lines," Applied Mathematics and Computation, vol. 241, pp. 214-221, 2014.

[18] P. Kayal and C. K. Chanda, "Placement of wind and solar based DGs in distribution system for power loss minimization and voltage stability improvement," International Journal Of Electrical Power \& Energy Systems, vol. 53, pp. 795-809, 2013.

[19] A. R. Di Fazio, et al., "Smart DER control for minimizing power losses in distribution feeders," Electric Power Systems Research, vol. 109, pp. 71-79, 2014.

[20] D.-L. Duan, et al., "Reconfiguration of distribution network for loss reduction and reliability improvement based on an enhanced genetic algorithm," International Journal Of Electrical Power \& Energy Systems, vol. 64, pp. 88-95, 2015.

[21] M. Moradi and M. Abedini, "A combination of genetic algorithm and particle swarm optimization for optimal DG location and sizing in distribution systems," International Journal Of Electrical Power \& Energy Systems, vol. 34, pp. 66-74, 2012.

[22] H. Bagheri Tolabi, et al., "Simultaneous Reconfiguration, Optimal Placement of DSTATCOM, and Photovoltaic Array in a Distribution System Based on Fuzzy-ACO Approach," IEEE Transaction on Sustainable Energy, vol. 6, pp. 210 - 218, 2015. 
[23] W. Sheng, et al., "Optimal power flow algorithm and analysis in distribution system considering distributed generation," IET Generation, Transmission \& Distribution, vol. 8, pp. 261-272, 2014.

[24] S. Alamelu, et al., "Optimal siting and sizing of UPFC using evolutionary algorithms," International Journal Of Electrical Power \& Energy Systems, vol. 69, pp. 222-231, 2015. 